with a previous audit from 2012; following which recommendations were made, including efforts to contact the source patients. Results A total of 126 patients attended for PEPSE during the 2014 audit period; median age 28 years (range 17-53); majority male (93.7\%); homosexual (81.0\%); White British (79.4\%). Baseline HIV tests were performed in 99.2\%; PEPSE was prescribed in accordance with BASHH recommendations in $98.4 \%$ and $97.6 \%$ were provided $<72 \mathrm{~h}$. In $15.1 \%$ the source was contacted.

In comparison with our 2012 audit, there were fewer women (6.3\% vs $20.6 \%)$ who accessed PEPSE and there was an improvement in PEPSE being prescribed in accordance with BASHH recommendations (98.4\% vs $92.7 \%)$. There was a statistically significant improvement in the number of source patients contacted $(15.1 \%$ vs $2.9 \%$; $<<0.01)$. In the case of 19 patients in whom the source was contacted, 4 were able to stop taking PEPSE (21.1\%).

Discussion/conclusion The number of patients accessing PEPSE has remained high and forms an important part of service provision in sexual health clinics. Contacting the source is an important step to reduce the unnecessary prescribing of PEPSE.

\section{P74 POST EXPOSURE PROPHYLAXIS: BASHH REGIONAL AUDIT 2014}

${ }^{1}$ Olivia Drew*, ${ }^{2}$ Emily Clarke, ${ }^{3}$ Alison Blume, ${ }^{4}$ Leela Sanmani, ${ }^{5}$ Neelam Radja, ${ }^{6}$ Sangeetha Sundaram, ${ }^{7}$ Cecilia Priestley. ${ }^{1}$ Royal Bournemouth Hospital NHS Trust, Bournemouth, UK; ${ }^{2}$ Solent NHS Trust, Southampton, UK; ${ }^{3}$ Solent NHS Trust, Portsmouth, UK; ${ }^{4}$ Solent NHS Trust, Winchester, UK; ${ }^{5}$ Solent NHS Trust, Basingstoke, UK; ${ }^{6}$ Salisbury NHS Trust, Salisbury, UK; 'Weymouth Community Hospital, Weymouth, UK

\subsection{6/sextrans-2015-052126.117}

Background/introduction In 2011 British Association of Sexual Health and HIV (BASHH) updated their guidelines on HIV post-exposure prophylaxis (PEP).

Aim(s)/objectives To audit the management of patients treated with PEP for both sexual and non-sexual risk in GUM clinics against BASHH PEP guidelines.

Methods A retrospective case notes review was performed on patients attending for PEP following both sexual and non-sexual risk, in 7 GUM clinics in Wessex between January-December 2013. Data collected included indication for PEP, time to commence, STI screening, completion rates and HIV testing done at baseline and 3 months post-PEP.

Results 98 case notes were reviewed. 77 patients had a sexual risk (47/77 men who have sex with men) and 21 a non-sexual risk. $92 \%$ of patients had a baseline HIV test at $<72 \mathrm{~h}$ (target 100\%). $73 \%$ of PEPSE prescriptions fitted within recommended indications, however only $28 \%$ of PEP prescriptions following non-sexual risk fitted within the recommended indication (target 90\%). 100\% of patients received PEP within $72 \mathrm{~h}$ and $62 \%$ of patients completed 4 weeks PEP (target $75 \%$ ). $54 \%$ of patients had an HIV test at 3 months post-PEP (target $60 \%$ ) and $70 \%$ of patients receiving PEPSE had an STI screen (target 90\%).

Conclusion This audit demonstrated some good management such as baseline HIV testing and the time to commence PEP. It also revealed areas to be improved, in particular PEP prescribing in a non-sexual risk situation, where often the risk was not a recommended indication. This highlights the importance of continued education to all PEP prescribers.
Category: HIV testing, new diagnoses and management

\section{P75 PATIENT SATISFACTION WITH HOME DELIVERY SERVICE FOR ANTIRETROVIRAL MEDICATION}

Michelle Penn, Janet Paterson, Arnold Fernandes, Kate Horn*. Royal United Hospital Bath NHS Foundation Trust, Bath, UK

\subsection{6/sextrans-2015-052126.118}

Background/introduction There is much interest in the use of home delivery services for antiretroviral medications. Advantages include convenience for patients, but notably, being VAT exempt, considerable cost benefits. In a small clinic like ours (cohort 174 patients), the estimated annual saving is $£ 85,000$. Disadvantages include concerns about confidentiality and inconvenience.

Aim(s)/objectives To assess the level of patient satisfaction with homecare delivery with a goal of $90 \%$, and to exclude 'never events': delivery to wrong person/address or patient running out of medication.

Methods Between April and July 2014 we conducted an opportunistic paper-based survey of patients attending the HIV clinic. Results were analysed using Microsoft excel.

Results Completed questionnaires were returned from 57\% of all homecare users. $85 \%$ reported telephone contact was good or very good but $23 \%$ experienced failure to deliver within the agreed time slot, some on multiple occasions. One patient reported running out of treatment and two deliveries had been made to an incorrect address. Overall satisfaction with the service was $81 \%$.

Discussion/conclusion This survey had a number of limitations: it was not completely randomised or anonymised, used subjective measures, did not account for patient compliance and did not explore reasons for declining homecare. Overall satisfaction with the service fell short of our goal of $90 \%$. More importantly there were 3 'never events', two of which involved potential breach of confidentiality and caused considerable distress to the patients. The results have been fed back to the homecare delivery provider with a particular focus on avoiding 'never events'.

\section{P76 STI SCREENING IN HIV POSITIVE PATIENTS ATTENDING A CITY-CENTRE HIV CLINIC}

Lisa Goodall*. SSOTP, Stoke on Trent, UK

\subsection{6/sextrans-2015-052126.119}

Background/introduction STI screening and treatment of HIV infected individuals is essential for the health of each individual and to prevent onward HIV transmission.

Aim(s)/objectives To audit STI screening among our HIV cohort against 2007 BHIVA, BASHH, and FSRH guidelines on management of SRH of people living with HIV.

Methods Case notes of the first 150 patients attending from 1 January 2014 were reviewed. Data gathered included: Demographics, sexual history taking in the last 6 months, STI screening in the last 12 months and STI diagnoses.

Results 54 patients were female (36\%) and 96 male (64\%). Average age was 43 (range 17-71). 81 patients (54\%) were White British, 53 (35\%) Black African. 95 (63\%) patients were heterosexual, 53 (35\%) gay, and $2(1 \%)$ bisexual. Demographics were representative of the whole cohort (444 patients). Sexual history was documented for 121 patients (81\%) in the last 